\title{
On a certain vector crank modulo 7
}

\author{
Michael D. Hirschhorn \\ School of Mathematics and Statistics \\ University of New South Wales \\ Sydney, NSW, 2052, Australia \\ m.hirschhorn@unsw.edu.au \\ Pee Choon Toh* \\ Mathematics \& Mathematics Education, \\ National Institute of Education, Nanyang Technological University \\ 1 Nanyang Walk, Singapore 637616 \\ peechoon.toh@nie.edu.sg
}

Submitted: Apr 25, 2014; Accepted: Jan 10, 2015; Published: Feb 9, 2015

Mathematics Subject Classifications: 11P83, 05A17

\begin{abstract}
We define a vector crank to provide a combinatorial interpretation for a certain Ramanujan type congruence modulo 7.
\end{abstract}

Keywords: partitions; congruences; crank

\section{Introduction}

In [7], one of the authors established several new Ramanujan type identities and congruences modulo 3, 5 and 7 for certain types of partition functions. For example, define $Q_{p o, \bar{p}}(n)$ as the number of partitions of $n$ into two colors, where the red colored parts form a partition into odd parts and the blue colored parts form an overpartition. Using the standard notation

$$
\begin{aligned}
& (a ; q)_{n}=\prod_{j=0}^{n-1}\left(1-a q^{j}\right) \\
& (a ; q)_{\infty}=\lim _{n \rightarrow \infty}(a ; q)_{n} \\
& \left(a_{1}, \ldots, a_{m} ; q\right)_{\infty}=\left(a_{1} ; q\right)_{\infty} \cdots\left(a_{m} ; q\right)_{\infty}
\end{aligned}
$$

\footnotetext{
*Supported by the NIE Academic Research Fund RI 3/12 TPC.
} 
for $|q|<1$ and $a, a_{1}, \ldots, a_{m} \neq 0$, we can write the generating function of $Q_{p o, \bar{p}}(n)$ as

$$
\sum_{n=0}^{\infty} Q_{p o, \bar{p}}(n) q^{n}=\frac{1}{\left(q ; q^{2}\right)_{\infty}} \times \frac{(-q ; q)_{\infty}}{(q ; q)_{\infty}}=\frac{(-q,-q ; q)_{\infty}}{(q ; q)_{\infty}}
$$

Toh [7] proved that

$$
\sum_{n=0}^{\infty} Q_{p o, \bar{p}}(7 n+2) q^{n} \equiv 0 \quad(\bmod 7) .
$$

Zhou [9] subsequently provided alternative proofs of all of the congruences in [7] with the exception of (1). She re-interpreted these partition functions as partitions into multicolors, introduced what she termed as multiranks - which are essentially vector cranks as defined by Garvan [4] - and proved that these vector cranks divided the partitions into equinumerous parts. The aim of this article is to define a vector crank that will explain (1) combinatorially.

\section{A vector crank}

If $\lambda$ is a partition, we define $\sigma(\lambda)$ and $n(\lambda)$ as the sum of the parts and the number of parts of $\lambda$ respectively. Let $\mathcal{D}, \mathcal{O}, \mathcal{P}$ denote the sets of partitions into distinct parts, partitions into odd parts, and unrestricted partitions respectively. Define the cartesian product

$$
\mathcal{V}=\mathcal{D} \times \mathcal{D} \times \mathcal{O} \times \mathcal{O} \times \mathcal{P} \times \mathcal{P}
$$

For a vector partition $\vec{\lambda}=\left(\lambda_{1}, \lambda_{2}, \lambda_{3}, \lambda_{4}, \lambda_{5}, \lambda_{6}\right) \in \mathcal{V}$ define a sum of parts $s$, a weight $w$ and a crank $r$ by

$$
\begin{aligned}
s(\vec{\lambda}) & =2 \sigma\left(\lambda_{1}\right)+\sigma\left(\lambda_{2}\right)+\sigma\left(\lambda_{3}\right)+\sigma\left(\lambda_{4}\right)+2 \sigma\left(\lambda_{5}\right)+2 \sigma\left(\lambda_{6}\right), \\
w(\vec{\lambda}) & =(-1)^{n\left(\lambda_{1}\right)} \\
r(\vec{\lambda}) & =2 n\left(\lambda_{3}\right)-2 n\left(\lambda_{4}\right)+n\left(\lambda_{5}\right)-n\left(\lambda_{6}\right) .
\end{aligned}
$$

The weighted count of vector partitions of $n$ with crank $m$, denoted by $N_{\mathcal{V}}(m, n)$, is given by

$$
N_{\mathcal{V}}(m, n)=\sum_{\substack{\vec{\lambda} \in \mathcal{V} \\ s(\vec{\lambda})=n \\ r(\vec{\lambda})=m}} w(\vec{\lambda})
$$

We also define the weighted count of vector partitions of $n$ with crank congruent to $k$ modulo $t$ by

$$
N_{\mathcal{V}}(k, t, n)=\sum_{m=-\infty}^{\infty} N_{\mathcal{V}}(m t+k, n)=\sum_{\substack{\vec{\lambda} \in \mathcal{V} \\ s(\vec{\lambda})=n \\ r(\vec{\lambda}) \equiv k(\bmod t)}} w(\vec{\lambda})
$$


Finally, we have the following generating function for $N_{\mathcal{V}}(m, n)$,

$$
\sum_{m=-\infty}^{\infty} \sum_{n=0}^{\infty} N_{\mathcal{V}}(m, n) z^{m} q^{n}=\frac{\left(q^{2} ; q^{2}\right)_{\infty}(-q ; q)_{\infty}}{\left(z^{2} q ; q^{2}\right)_{\infty}\left(z^{-2} q ; q^{2}\right)_{\infty}\left(z q^{2} ; q^{2}\right)_{\infty}\left(z^{-1} q^{2} ; q^{2}\right)_{\infty}} .
$$

Theorem 1. The following equation holds for all nonnegative integers $n$.

$$
N_{\mathcal{V}}(0,7,7 n+2)=N_{\mathcal{V}}(1,7,7 n+2)=\cdots=N_{\mathcal{V}}(6,7,7 n+2)=\frac{Q_{p o, \bar{p}}(7 n+2)}{7} .
$$

The main ingredient in the proof of the theorem is Winquist's identity [8], which is a variant of the $B_{2}$ case of the Macdonald identities [5]. We state the identity in the following symmetric form [6, Eq. (3.1)]. If we define

$$
\begin{aligned}
& F_{1}(x)=\sum_{j=-\infty}^{\infty}(-1)^{j} q^{3 j^{2}}\left(x^{3 j}+x^{-3 j}\right), \\
& F_{2}(x)=\sum_{k=-\infty}^{\infty}(-1)^{k} q^{3 k^{2}+2 k}\left(x^{3 k+1}+x^{-3 k-1}\right),
\end{aligned}
$$

we have

$$
F_{1}(x) F_{2}(y)-F_{1}(y) F_{2}(x)=-\frac{2}{x}\left(x q, \frac{q}{x}, y q, \frac{q}{y}, x y, \frac{q^{2}}{x y}, \frac{x}{y}, \frac{y q^{2}}{x}, q^{2}, q^{2} ; q^{2}\right)_{\infty} .
$$

Proof of Theorem 1. If we set $\zeta=\exp (2 \pi i / 7)$ in (5), we obtain

$$
\begin{aligned}
& \sum_{t=0}^{6} \zeta^{t} \sum_{n=0}^{\infty} N_{\mathcal{V}}(t, 7, n) q^{n} \\
& =\sum_{m=-\infty}^{\infty} \sum_{n=0}^{\infty} N_{\mathcal{V}}(m, n) \zeta^{m} q^{n} \\
& =\frac{\left(q^{2} ; q^{2}\right)_{\infty}}{\left(q, \zeta^{2} q, q / \zeta^{2}, \zeta q^{2}, q^{2} / \zeta ; q^{2}\right)_{\infty}} \\
& =\frac{\left(\zeta q, q / \zeta, \zeta^{3} q, q / \zeta^{3} ; q^{2}\right)_{\infty}}{\left(q^{7} ; q^{14}\right)_{\infty}} \times \frac{\left(q^{2}, q^{2}, \zeta^{2} q^{2}, q^{2} / \zeta^{2}, \zeta^{3} q^{2}, q^{2} / \zeta^{3} ; q^{2}\right)_{\infty}}{\left(q^{14} ; q^{14}\right)_{\infty}} \\
& =\frac{F_{1}\left(\zeta^{3}\right) F_{2}(\zeta)-F_{1}(\zeta) F_{2}\left(\zeta^{3}\right)}{2 \zeta\left(1-\zeta^{2}\right)\left(1-\zeta^{3}\right)\left(q^{7} ; q^{7}\right)_{\infty}},
\end{aligned}
$$

where we used (6c) with $x=\zeta^{3}$ and $y=\zeta$.

Since $3 j^{2} \equiv 0,3,5,6(\bmod 7)$ and $3 k^{2}+2 k \equiv 0,1,2,5(\bmod 7)$, the power of $q$ in $q^{3 j^{2}+3 k^{2}+2 k}$ is congruent to 2 modulo 7 exactly when $j \equiv 0(\bmod 7)$ and $k \equiv 2(\bmod 7)$. This means that the coefficient of $q^{7 n+2}$ in

$$
F_{1}\left(\zeta^{3}\right) F_{2}(\zeta)-F_{1}(\zeta) F_{2}\left(\zeta^{3}\right)
$$


is zero since

$$
(-1)^{j+k}\left(\zeta^{9 j}+\zeta^{-9 j}\right)\left(\zeta^{3 k+1}+\zeta^{-3 k-1}\right)-(-1)^{j+k}\left(\zeta^{3 j}+\zeta^{-3 j}\right)\left(\zeta^{9 k+3}+\zeta^{-9 k-3}\right)=0
$$

when $j \equiv 0(\bmod 7)$ and $k \equiv 2(\bmod 7)$. Thus

$$
\sum_{t=0}^{6} N_{\mathcal{V}}(t, 7,7 n+2) \zeta^{t}=0
$$

Since the minimal polynomial for $\zeta$ over the rational numbers is

$$
p(x)=1+x+x^{2}+\cdots+x^{6},
$$

we conclude that

$$
N_{\mathcal{V}}(0,7,7 n+2)=N_{\mathcal{V}}(1,7,7 n+2)=\cdots=N_{\mathcal{V}}(6,7,7 n+2) .
$$

We end by indicating how one may prove (1) directly as the details were omitted in [7]. This can be done by observing that

$$
\begin{aligned}
\sum_{n=0}^{\infty} Q_{p o, \bar{p}}(n) q^{n} & =\frac{\left(q^{2} ; q^{2}\right)_{\infty}^{2}}{(q ; q)_{\infty}^{3}} \\
& \equiv \frac{\left(q^{2} ; q^{2}\right)_{\infty}^{9}}{(q ; q)_{\infty}^{3}} \times \frac{1}{\left(q^{14} ; q^{14}\right)_{\infty}} \quad(\bmod 7)
\end{aligned}
$$

Thus (1) is equivalent to proving the coefficients of $q^{7 n+2}$ in

$$
\frac{\left(q^{2} ; q^{2}\right)_{\infty}^{9}}{(q ; q)_{\infty}^{3}}
$$

are all divisible by 7 . We offer three alternative ways of doing this. The easiest way is to appeal directly to [3, Th. 2]. Alternatively, we can use one of the Macdonald identities associated with the $C_{2}^{\vee}$ root system [5, p. 137] or [6, Eq. 3.12], to express

$$
\frac{\left(q^{2} ; q^{2}\right)_{\infty}^{9}}{(q ; q)_{\infty}^{3}}=\sum_{\substack{\alpha \equiv 1 \\ \beta \equiv 3}} \frac{1}{(\bmod 8)} 8\left(\beta^{2}-\alpha^{2}\right) q^{\frac{\alpha^{2}+\beta^{2}-10}{16}}
$$

If the exponent of $q$ is congruent to 2 modulo 7 , we have

$$
\alpha^{2}+\beta^{2} \equiv 16(2)+10 \equiv 0 \quad(\bmod 7)
$$

Since -1 is a quadratic nonresidue modulo 7,7 must divide both $\alpha$ and $\beta$. The third way is to apply the Hecke operator $T_{7}$ to $\frac{\eta(16 \tau)^{9}}{\eta(8 \tau)^{3}}$, a weight 3 cusp form of level 128 . One can refer to [1] for examples of how this may be done. 


\section{Acknowledgements}

We thank the anonymous referee for helpful comments that improved the article. In particular, the referee pointed out that the coefficients $N_{\mathcal{V}}(m, n)$ are nonnegative which can be proved using the following corollary [2, Eq. (4.1)] of the $q$-binomial theorem.

Proposition 2. If $|q|,|t|<1$ then

$$
\frac{(a t ; q)_{\infty}}{(a ; q)_{\infty}(t ; q)_{\infty}}=\frac{1}{(a ; q)_{\infty}}+\sum_{n=1}^{\infty} \frac{t^{n}}{\left(a q^{n} ; q\right)_{\infty}(q ; q)_{n}} .
$$

\section{References}

[1] S. Ahlgren. Multiplicative relations in powers of Euler's product. J. Number Theory, 89:222-233, 2001.

[2] A. Berkovich, and F. G. Garvan. K. Saito's Conjecture for nonnegative eta products and analogous results for other infinite products. J. Number Theory, 128:1731-1748, 2008.

[3] S. Cooper, M. D. Hirschhorn, and R. Lewis. Powers of Euler's product and related identities. Ramanujan J., 4:137-155, 2000.

[4] F. G. Garvan. New combinatorial interpretations of Ramanujan's partition congruences mod 5, 7 and 11. Trans. Amer. Math. Soc., 305:47-77, 1988.

[5] I. G. Macdonald. Affine root systems and Dedekind's $\eta$-function. Invent. Math., 15:91-143, 1972.

[6] P. C. Toh. Generalized $m$ th order Jacobi theta functions and the Macdonald identities. Int. J. Number Theory, 4:461-474, 2008.

[7] P. C. Toh. Ramanujan type identities and congruences for partition pairs. Discrete Math., 312:1244-1250, 2012.

[8] L. Winquist. An elementary proof of $p(11 m+6) \equiv 0(\bmod 11)$. J. Combin. Theory, 6:56-59, 1969.

[9] R. R. Zhou. Multiranks for partitions into multi-colors. Electron. J. Combin., 19:\#P8, 2012. 